\section{Uso de medicamentos contínuos \\ e fatores associados em idosos de Quixadá, Ceará}

\section{Continuous-use medication and associated factors in the elderly living in Quixadá, Ceará, Brazil}

\section{Gilmar de Oliveira Barros Silva'}

Ana Paula Soares Gondim'

Mirian Parente Monteiro',"11

Mirna Albuquerque Frota'

\section{André Luis Lima de Meneses,"11!}

' Mestrado em Saúde Coletiva da Universidade de Fortaleza. Fortaleza, CE, Brasil.

" Programa de Pós-Graduação em Ciências Farmacêuticas da Universidade Federal do Ceará.

I"' Pós-Graduação em Atenção Farmacêutica da Universidade de Fortaleza.

Apoio financeiro: Bolsa de Mestrado da Fundação Cearense de Apoio ao Desenvolvimento Científico e Tecnológico (FUNCAP-CE). BMD-0008-00108.01.08.

Correspondência: Ana Paula Soares Gondim. Rua Cleiton Helaide Pimenta, 815, casa 02, Jardim das Oliveiras - Fortaleza - CE - CEP 60.821-410. E-mail: anapaulasgondim@unifor.br

\section{Resumo}

Objetivo: Analisar a polifarmácia (uso diário de dois ou mais) de medicamentos contínuos e seus fatores associados em idosos. Métodos: Estudo seccional realizado com idosos residentes em área urbana do município de Quixadá-CE, no período de maio a dezembro de 2009. A amostra compôs-se de 384 indivíduos, com 60 anos ou mais, usuários de medicamentos contínuos. As variáveis estudadas foram as sociodemográficas, econômicas e das características de saúde e referentes ao uso de medicamentos. Para a análise da associação entre a variável dependente polifarmácia de medicamentos contínuos e as variáveis independentes foi elaborado um modelo de regressão logística. Resultados: Os resultados mostram predominância de idosos do sexo feminino, faixa etária entre 60 e 69 anos, casados, ensino fundamental incompleto, sem exercer atividade remunerada, renda familiar de até um salário mínimo, habitando em moradia própria, residindo com até três pessoas. Constatou-se uma prevalência de $70,6 \%$ de polifarmácia em idosos, sendo mais elevada no sexo feminino $(66,4 \%)$. Os fatores associados positivamente ao uso de dois ou mais medicamentos contínuos foram: renda familiar acima de um salário mínimo (OR 2,83; IC95\% = 1,54-5,32); duas ou mais condições crônicas autorreferidas (OR 17,71; IC95\% = 9,80-31,990) e autopercepção da qualidade de vida regular e ruim (OR 2,85; IC95\% = 1,60-5,07). Conclusões: Constatou-se uma prática de polifarmácia de medicamentos contínuos em idosos com renda familiar superior a um salário mínimo, que apresenta duas ou mais condições crônicas e autopercepção da sua qualidade de vida entre regular e ruim. Situação que remete a questões relativas aos aspectos social, cultural, econômico e de saúde.

Palavras-chave: Idoso. Saúde do idoso. Uso de medicamentos. Farmacoepidemiologia. Fatores epidemiológicos. Estudos transversais. 


\section{Abstract}

Objective: To analyze polypharmacy (daily use of two or more) of continuous use medication and its associated factors in the elderly. Methods: A cross sectional study conducted with elderly individuals living in an urban area of the municipality of Quixadá, Ceara, Brazil from May to December, 2009. The sample consisted of 384 individuals who were 60 years old or older and users of continuous medication. The variables studied were sociodemographic, economical, and health characteristics related to use of medications. For the analysis of the association between the dependent variable polypharmacy of continuous-use medication and the independent variables a logistic regression model was developed. Results: Results showed a predominance of females, between 60 and 69 years old, married, with incomplete elementary school education, non-paid job, family income of one minimum salary, living in their own home and together with up to three individuals. A prevalence of $70.6 \%$ of polypharmacy in the elderly was found, and it was higher in women (66.4\%). Factors positively associated with the use of two or more continuous-use medication were: family income over one minimum salary (OR 2.83; CI95\% = 1.54-5.32); two or more self-reported chronic conditions (OR 17.71; CI95\% $=9.80-31.990)$ and self-perception of regular to poor quality of life (OR 2.85; CI95\% $=1 \cdot 60-5 \cdot 07)$. Conclusions: The study observed a practice of polypharmacy of continuous-use medications in the elderly with family income over one minimum wage, two or more chronic conditions and self-perception of regular to poor quality of life. Such status leads to questions on social, cultural, economic and health aspects.

Keywords: Elderly. Elderly health. Medication use. Pharmacoepidemiology. Epidemiological factors. Cross-sectional studies.

\section{Introdução}

O envelhecimento populacional não se refere nem a indivíduos, nem a cada geração, mas sim à mudança na estrutura etária da população, o que produz um aumento do peso relativo das pessoas acima de determinada idade ${ }^{1}$. Em 2025, em muitos países em desenvolvimento, particularmente na América Latina e Ásia, o aumento da população idosa será de 300\%. Em 2050, haverá dois bilhões de pessoas com idade acima de 60 anos, $80 \%$ destas vivendo em países em desenvolvimento ${ }^{2}$.

Para a Organização Mundial da Saúde, o desafio do envelhecimento populacional é o crescimento das doenças crônicas e de suas incapacidades, bem como o status de dependência ou de deficiência do idoso, além disso, esse crescimento gera demanda por serviços de saúde ${ }^{3,4}$. A presença de condições crônicas tornou-se uma epidemia. Nos Estados Unidos, em 2004, mais da metade da população, aproximadamente 133 milhões de americanos, viviam pelo menos com uma condição crônica ${ }^{5}$. No Brasil, segundo os dados da Pesquisa Nacional por Amostra de Domicílios, realizada em 2009, $77,4 \%$ dos idosos brasileiros declararam ter pelo menos uma doença crônica ${ }^{6}$.

Os idosos constituem o grupo etário mais medicalizado, desenvolvendo a prática da polifarmácia. Além disso, este grupo apresenta os maiores indicadores de prevalência e incidência de comorbidades? . Há particularidades farmacodinâmicas e farmacocinéticas entre os idosos que os tornam mais vulneráveis a eventos adversos e interações medicamentosas ${ }^{8}$.

Entende-se por polifarmácia a utilização por um mesmo paciente de dois ou mais medicamentos (prescritos ou não). A polifarmácia em idosos tem sido identificada como o principal fator relacionado com a segurança dos medicamentos no tocante a ocorrência de reações adversas e interações medicamentosas. Existe mais de uma maneira de conceituar polifarmácia, conforme a abordagem qualitativa ou quantitativa. A abordagem qualitativa observa o uso 
de um ou mais fármacos desnecessários ao esquema terapêutico, ou seja, o uso a mais de um ou mais fármacos além do que está clinicamente indicado. A abordagem quantitativa considera apenas o número de fármacos utilizados por um determinado indivíduo, independente da necessidade clínica, variando desde o consumo de dois até cinco ou mais fármacos ${ }^{10,11}$.

$\mathrm{O}$ uso indiscriminado e inadequado de medicamentos em idosos constitui hoje um sério problema de Saúde Coletiva, no qual a vulnerabilidade dos idosos aos eventos adversos a medicamentos é bastante alta ${ }^{12}$.

Nos últimos sete anos, vários estudos farmacoepidemiológicos em idosos foram publicados no Brasil ${ }^{7,13,14}$. Alguns desses estudos enfatizam as populações das regiões metropolitanas do País ${ }^{7,14}$, sendo necessário desenvolver inquéritos populacionais que relacionam a prevalência do uso de medicamentos contínuos e polifarmácia em idosos em outras regiões, particularmente no semiárido do Nordeste. Esta região do país tem recebido pouca atenção quanto à realização de estudos em vários âmbitos das ciências, particularmente na área da Saúde Coletiva.

O presente estudo teve como objetivo analisar a polifarmácia (uso diário de dois ou mais) de medicamentos contínuos e seus fatores associados em idosos em um município do Nordeste Brasileiro.

\section{Métodos}

Estudo seccional com idosos residentes em área urbana do município de Quixadá, Estado do Ceará, realizado no período de maio a dezembro de 2009. O município de Quixadá está situado na Região Central do Estado, localizado na zona do semiárido nordestino, distante $165 \mathrm{~km}$ da capital, Fortaleza. Em 2004, Quixadá possuía uma população de 69.654 habitantes, com 46.888 habitantes residindo na área urbana, com taxa de urbanização de $67,3 \%$, densidade demográfica de 33,7 hab. $/ \mathrm{km}^{2}$ e esperança de vida ao nascer de 69,6 anos ${ }^{15}$.

Para o cálculo da amostra foram considerados: a população-alvo do estudo de
6.910 idosos com 60 anos ou mais de idade e residentes na área urbana de Quixadá ${ }^{15}$, proporção de $50 \%$ de idosos que fazem uso de medicamentos contínuos, erro amostral relativo de $5 \%$ e nível de significância de $5 \%$, compreendendo assim uma amostra aleatória simples de 384 indivíduos.

A seleção dos idosos foi feita por amostragem estratificada proporcional entre os 21 bairros que compõem a área urbana de Quixadá. Foi realizado um cálculo proporcional de $10 \%$ de idosos em cada bairro, e em seguida foi realizado um sorteio da rua e do número do domicílio. O critério de inclusão do idoso foi usar medicamentos contínuos nos últimos seis meses.

Aplicou-se um formulário pré-codificado e validado para coleta de dados das características sociodemográficas e econômicas, características de saúde, de qualidade de vida e de uso de medicamentos contínuos. Este instrumento foi aplicado por entrevistadores previamente treinados e supervisionados pelo pesquisador responsável pelo trabalho de campo.

As variáveis estudadas foram: variável dependente (uso da polifarmácia de medicamentos contínuos) e variáveis independentes (sexo, idade, estado conjugal, escolaridade, renda familiar, condição de moradia, coabitação, participação em atividade de grupo, prática de atividade física, condições crônicas não transmissíveis autorreferidas, número de comorbidades autorreferidas, autoavaliação de saúde e autopercepção da qualidade de vida e aquisição dos medicamentos). A variável polifarmácia é definida como uso de dois ou mais medicamentos contínuos (prescritos ou não). A participação em atividade de grupo é definida por participar de associação comunitária, sindicato, grupo religioso e grupo de idosos. Dez condições crônicas não transmissíveis autorreferidas foram descritas (hipertensão, diabetes, acidente vascular cerebral, osteoporose, dislipidemias, doença coronariana, obesidade, doença pulmonar obstrutiva crônica, depressão e câncer). A pergunta "Você está satisfeito(a) com a sua saúde?" produziu a variável autoavaliação de saúde, 
enquanto a pergunta "Como você avalia sua qualidade de vida?” gerou a variável autopercepção da qualidade de vida.

As variáveis relacionadas ao uso de medicamentos contínuos foram: grupo terapêutico, indicação médica, aquisição dos medicamentos, motivos do uso e não uso de medicamentos, medicamentos genéricos e medicamentos potencialmente inapropriados para a prescrição em idosos. Os medicamentos foram identificados nos domicílios dos idosos, de acordo com a sua apresentação ou prescrição médica. Os grupos terapêuticos dos medicamentos foram classificados de acordo com a Classificação Anatomical Therapeutic Chemical (ATC) ${ }^{16}$, descritos pelo primeiro e quinto níveis terapêuticos. Os medicamentos potencialmente inapropriados para idosos foram os medicamentos que deveriam ser evitados, independente da dose, duração do tratamento ou circunstâncias clínicas, e que, segundo os critérios Beers-Fick, traziam riscos desnecessários para os idosos ${ }^{17}$.

Os dados foram armazenados no programa de computação para análise estatística SPSS 14.0 para Windows e analisados no programa estatístico Stata, versão 11.0. Para se avaliar as diferenças estatísticas foram aplicados o teste Qui-Quadrado de Pearson $\left(\chi^{2}\right)$ ou o teste Exato de Fischer, levando-se em consideração o tamanho da amostra como condição para aplicação dos respectivos testes. Elaborou-se um modelo de regressão logística para analisar a associação entre a ocorrência de polifarmácia e as variáveis independentes, considerando intervalo de confiança de $95 \%$ e nível de significância de $5 \%$.

O projeto foi aprovado pelo Comitê de Ética em Pesquisa da Universidade de Fortaleza (Protocolo № 073/2009) e todos os participantes assinaram termo de consentimento livre e esclarecido, de acordo com a Resolução no 196/96 ${ }^{18}$.

\section{Resultados}

Dos 384 idosos elegíveis para o estudo, todos responderam ao formulário, significando uma taxa de resposta de $100 \%$. As características predominantes foram: sexo feminino $(64,3 \%)$, idade entre 60 e 69 anos $(41,0 \%)$ e média de $72( \pm 8)$ anos, casados $(56,0 \%)$, ensino fundamental incompleto $(54,4 \%)$, não realiza atividade remunerada $(84,1 \%)$, renda familiar de até um salário mínimo $(66,1 \%)$, residentes em moradia própria $(85,2 \%)$ e morando com até três pessoas (63,5\%). Quase todo o arranjo familiar era composto por familiares $(97,1 \%)$, destacando-se cônjuge e filhos $(39,3 \%)$. A maioria não participava de atividade em de grupo $(78,1 \%)$ e $26,3 \%$ dos idosos realizavam atividade física habitual. Entre os idosos, foi observado que 44,3\% estavam satisfeitos com a saúde e 43,7\% consideravam positivamente sua qualidade de vida.

O número de condições crônicas não transmissíveis autorreferidas pelos idosos variou entre uma e cinco, com média de duas $( \pm 1)$ condições; a maioria $(59,9 \%)$ possuía mais de duas condições crônicas; e não foi observada ausência de condição crônica. Quanto à distribuição dessas condições crônicas, evidenciou-se a "hipertensão" como a mais frequente $(41,9 \%)$, seguida de “diabetes" (13,0\%) e "dislipidemia” (8,5\%), compondo quase $50,0 \%$ das condições cardiovasculares e metabólicas (Figura 1).

A prevalência da polifarmácia de medicamentos contínuos foi de $70,6 \%$ no grupo estudado, sendo mais elevada no sexo feminino $(66,4 \%)$ em relação ao sexo masculino $(33,6 \%)$. A faixa etária mais prevalente $(63,8 \%)$ foi a faixa acima de 70 anos e $36,2 \%$ dos idosos tinham entre 60 e 69 anos de idade.

A Tabela 1 apresenta a prevalência da polifarmácia de medicamentos contínuos em idosos, segundo as características sociodemográficas, econômicas e das condições de saúde. Na análise bivariada, foram observadas diferenças estatisticamente significativas entre a variável polifarmácia e as variáveis faixa etária acima de 70 anos de idade ( $\mathrm{p}=0,001)$, sem escolaridade $(\mathrm{p}=0,035)$, renda familiar mais de um salário mínimo ( $p=0,008)$, com duas ou mais condições 


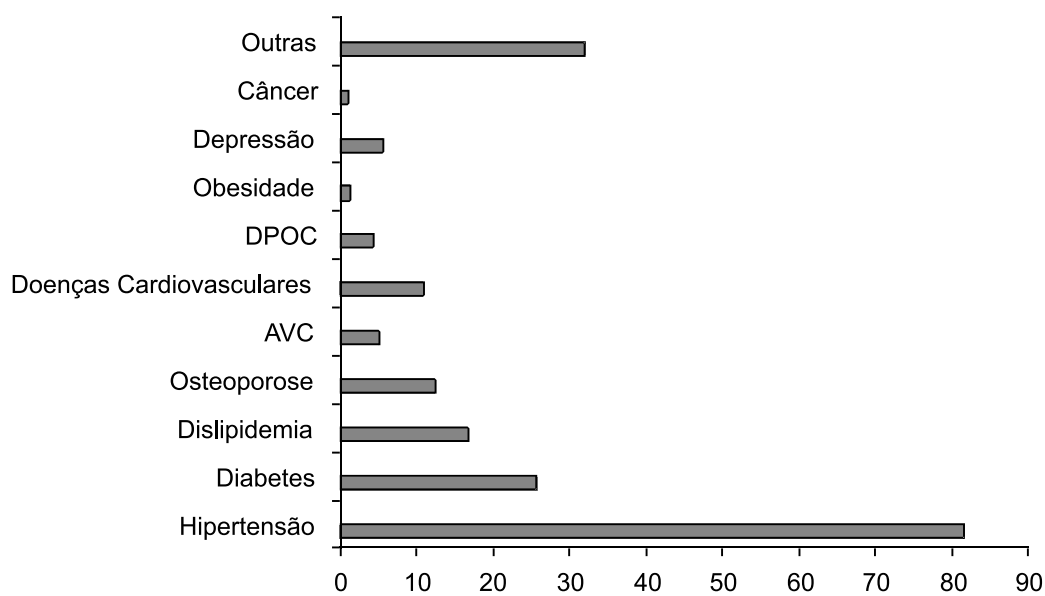

Figura 1 - Condições crônicas não transmissíveis autorreferidas pelos idosos usuários de medicamentos contínuos. Município de Quixadá, CE, 2009.

Figure 1 - Non-communicable chronic conditions self-reported by the elderly who use continuoususe medication. Municipality of Quixadá, CE, 2009.

crônicas não transmissíveis autorreferidas ( $\mathrm{p}=0,001)$, aquisição de medicamentos em farmácias comunitárias privadas ou populares ( $p=0,001)$, autoavaliação de saúde muito insatisfeita e insatisfeita $(\mathrm{p}=0,001) \mathrm{e}$ autopercepção da qualidade de vida regular e ruim $(\mathrm{p}=0,017)$.

O número de medicamentos usados pelos idosos variou entre um e dez medicamentos, com média de 2,4 medicamentos/ idoso. Quando se estratificou por sexo, observou-se uma média mais elevada no sexo feminino (2,5 medicamentos/idoso), enquanto no sexo masculino foi de 2,2 medicamentos/idoso. A faixa etária de 70 a 79 anos apresentou uma média mais elevada de uso de medicamentos de 2,6, na faixa acima de 80 anos de idade a média foi de 2,4 medicamentos/idoso e na faixa entre 60 e 69 anos foi de 2,1 medicamentos/idoso.

Foram identificadas 139 substâncias farmacológicas diferentes, de acordo com a classificação ATC, resultando em um total de 910 medicamentos utilizados pelos idosos. A frequência mais observada, segundo o primeiro nível terapêutico, foi o sistema cardiovascular $(56,7 \%)$, seguido por trato alimentar e metabolismo (17,5\%) e sistema nervoso (11,0\%). Na distribuição por medicamentos, o captopril foi o mais informado $(17,5 \%)$, seguido por hidroclorotiazida
$(13,1 \%)$, glibenclamida $(8,4 \%)$ e cloridrato de propranolol $(6,2 \%)$.

$\mathrm{O}$ uso de medicamentos genéricos entre os idosos obteve uma proporção de $33,9 \%$, sendo observada maior proporção entre as mulheres $(35,2 \%)$, enquanto entre os homens foi de $31,4 \%$. Quase todos os idosos usavam medicamentos de origem alopática e oriundos de prescrição médica. A aquisição dos medicamentos mais mencionada por eles foi a distribuição gratuita na farmácia comunitária do SUS $(74,5 \%)$, seguida da compra em farmácias populares $(12,2 \%)$ e em farmácias privadas $(9,4 \%)$. Metade dos idosos relatou ter dificuldade em adquirir gratuitamente os medicamentos de uso contínuo, sendo o motivo mais referido a falta desses medicamentos na rede assistencial do SUS (87,6\%).

Verificou-se uma proporção de 9,5\% do uso de medicamentos potencialmente inapropriados entre os idosos. Os benzodiazepínicos de meia-vida longa $(2,6 \%)$ foram os medicamentos que apresentaram maior proporção, seguidos dos antidepressivos tricíclicos $(1,3 \%)$ e dos glicosídeos cardiotônicos (1,3\%).

A análise bivariada mostrou uma associação positiva entre a polifarmácia de medicamentos contínuos a idade acima de 70 anos de idade (OR 2,14; IC95\% = 1,34-3,43); 
Tabela 1 - Prevalência da polifarmácia de medicamentos contínuos em idosos, segundo as variáveis sociodemográficas, econômicas e das condições de saúde. Município de Quixadá, $\mathrm{CE}$, 2009.

Table 1 - Prevalence of polypharmacy of continuous-use medication in the elderly according to sociodemographic and economic variables and health conditions. Municipality of Quixadá, CE, 2009.

\begin{tabular}{|c|c|c|c|}
\hline Variáveis & $\mathbf{n}$ & $\%$ & $\mathbf{p}$ \\
\hline Sexo & & & 0,184 \\
\hline Feminino & 247 & 72,9 & \\
\hline Masculino & 137 & 66,4 & \\
\hline Faixa etária (anos) & & & 0,001 \\
\hline $60-69$ & 160 & 61,3 & \\
\hline$\geq 70$ & 224 & 77,2 & \\
\hline Situação conjugal & & & 0,400 \\
\hline Casado & 215 & 68,8 & \\
\hline Solteiro & 169 & 72,8 & \\
\hline Escolaridade & & & 0,035 \\
\hline Não & 143 & 76,9 & \\
\hline Sim & 241 & 66,8 & \\
\hline Atividade remunerada & & & 0,748 \\
\hline Não & 323 & 70,9 & \\
\hline Sim & 61 & 68,9 & \\
\hline Renda familiar* & & & 0,008 \\
\hline Até um salário & 254 & 66,1 & \\
\hline Mais de um salário & 130 & 79,2 & \\
\hline Tipo de moradia & & & 0,100 \\
\hline Própria & 327 & 72,2 & \\
\hline Não própria & 57 & 61,4 & \\
\hline Quantidade de pessoas que mora & & & 0,514 \\
\hline Até três pessoas & 244 & 71,7 & \\
\hline Acima de três pessoas & 140 & 68,6 & \\
\hline Arranjo familiar & & & 0,608 \\
\hline Familiares & 373 & 70,8 & \\
\hline Sozinho & 11 & 63,6 & \\
\hline Atividade de grupo & & & 0,461 \\
\hline Não & 300 & 69,7 & \\
\hline Sim & 84 & 73,8 & \\
\hline Condições crônicas** & & & 0,001 \\
\hline Uma condição & 154 & 40,3 & \\
\hline Duas ou mais condições & 230 & 90,9 & \\
\hline Aquisição de medicamentos & & & 0,001 \\
\hline Farmácias exclusivas do SUS & 198 & 63,1 & \\
\hline Farmácias populares e privadas & 186 & 78,5 & \\
\hline Prática de atividade física habitual & & & 0,276 \\
\hline Não & 283 & 72,1 & \\
\hline Sim & 101 & 66,3 & \\
\hline Autoavaliação de saúde & & & 0,001 \\
\hline Muito satisfeito/satisfeito & 170 & 61,8 & \\
\hline Muito insatisfeito/insatisfeito & 214 & 77,6 & \\
\hline Autopercepção da qualidade de vida & & & 0,017 \\
\hline Boa/ótima & 168 & 64,3 & \\
\hline Regular/ruim & 216 & 75,5 & \\
\hline
\end{tabular}


Tabela 2 - Variáveis com possibilidade* de associação à polifarmácia de medicamentos contínuos em idosos. Município de Quixadá, CE, 2009.

Table 2 - Variables with possibility* of association with polypharmacy of continuous-use medication in the elderly. Municipality of Quixadá, CE, 2009.

\begin{tabular}{lccc}
\hline Variáveis & $\begin{array}{c}\text { OR Não } \\
\text { ajustada }\end{array}$ & IC95\% & $\mathrm{p}$ \\
\hline Idade acima de 70 anos & 2,14 & $1,34-3,43$ & 0,007 \\
Sem escolaridade & 1,65 & $1,01-2,74$ & 0,035 \\
Renda familiar mais de um salário & 1,95 & $1,16-3,34$ & 0,008 \\
Duas ou mais condições crônicas & 14,77 & $8,25-26,90$ & $<0,001$ \\
Aquisição de medicamentos em farmácias populares e privadas & 2,13 & $1,32-3,45$ & 0,001 \\
Autoavaliação de saúde muito insatisfeito e insatisfeito & 2,14 & $1,33-3,43$ & $<0,001$ \\
Autopercepção da qualidade de vida regular e ruim & 1,70 & $1,07-2,72$ & 0.017 \\
\hline
\end{tabular}

*Selecionados para posterior análise de regressão logística com valor $p<0,05$.

*Selected for posterior logistic regression analysis with $p<0.05$ value.

Tabela 3 - Variáveis do modelo de regressão logística associado à polifarmácia de medicamentos contínuos em idosos. Município de Quixadá, CE, 2009.

Table 3 - Logistic regression model variables associated with polypharmacy of continuou-uses medication in the elderly. Municipality of Quixadá, CE, 2009.

\begin{tabular}{lccc}
\hline Variáveis & OR Ajustada & IC95\% & $\mathrm{p}$ \\
\hline Renda familiar mais de um salário & 2,83 & $1,54-5,32$ & 0,001 \\
Duas ou mais condições crônicas & 17,71 & $9,80-31,99$ & $<0,001$ \\
Autopercepção da qualidade de vida regular e ruim & 2,85 & $1,60-5,07$ & $<0,001$ \\
\hline
\end{tabular}

sem escolaridade (OR 1,65; IC95\% = 1,012,74); renda familiar de mais de um salário mínimo (OR 1,95; IC95\% = 1,16-3,34); com duas ou mais condições crônicas autorreferidas (OR 14,77; IC95\% = 8,25-26,90); aquisição de medicamentos em farmácias comunitárias privadas ou populares (OR 2,13; IC95\% = 1,32-3,45); autoavaliação de saúde muito insatisfeita e insatisfeita (OR 2,$14 ; \mathrm{IC} 95 \%=1,33-3,43$ ) e autopercepção da qualidade de vida regular e ruim (OR 1,70; IC95\% $=1,07-2,72$ ) (Tabela 2).

No modelo de regressão logística observou-se que estavam associados positivamente à polifarmácia de medicamentos contínuos, as variáveis renda familiar mais de um salário mínimo (OR 2,83; IC95\% = 1,54-5,32); com duas ou mais condições crônicas autorreferidas (OR 17,71; IC95\% = 9,80-31,99) e autopercepção da qualidade de vida regular e ruim (OR 2,85; IC95\% = 1,60-5,07) (Tabela 3).

\section{Discussão}

Neste estudo observou-se uma alta prevalência da polifarmácia de $70,6 \%$ de medicamentos contínuos em idosos em um município do semiárido do Nordeste Brasileiro. Os fatores associados positivamente a esse fato foram renda familiar mais de um salário mínimo; com duas ou mais condições crônicas autorreferidas e autopercepção da qualidade de vida regular e ruim.

A prevalência da polifarmácia do uso de medicamentos contínuos em idosos encontrada neste estudo foi superior ao observado em alguns estudos com populações acima de 100.000 habitantes ${ }^{19,20}$. Apesar de não ter sido alvo estudar população idosa extensa, este estudo se propôs analisar a polifarmácia de medicamentos contínuos em um município da região do semiárido do Nordeste Brasileiro com menos de 100.000 habitantes. 
Outros estudos indicaram que as características sociodemográficas e as condições de saúde, como renda, escolaridade, arranjos familiares multigeracionais e a quantidade de condições crônicas são fatores associados ao uso de medicamentos contínuos em idosos ${ }^{13,21,22}$.

Assim como em alguns estudos realizados no Brasil, este estudo também verificou uma maior frequência da polifarmácia no sexo feminino ${ }^{23}$, com predominância de renda superior a dois salários mínimos e baixa escolaridade ${ }^{14,24}$.

A alta proporção de hipertensão e diabetes autorreferidas confirma os achados da literatura ${ }^{25,26}$. Considera-se um desafio para as políticas públicas, principalmente no campo da Saúde Coletiva, propor a adoção de estratégias de promoção da saúde e reorganização dos serviços voltados para essas condições crônicas não transmissíveis.

O estudo observou uma baixa frequência de práticas de atividade física habitual entre os idosos, devendo-se considerar que as estratégias de promoção da saúde, voltadas à incorporação dessas práticas no cotidiano da população idosa, podem reduzir o impacto das doenças crônicas no perfil geral de morbimortalidade brasileiro e melhorar a autoavaliação da qualidade de vida.

A expressiva baixa frequência de atividade em grupo é um fator importante de vulnerabilidade neste estudo. Dado que a participação de atividades em grupo, principalmente aquelas voltadas à promoção da saúde e ao estilo de vida saudável, podem contribuir para superação do modelo biomédico ${ }^{12}$.

A média do uso de medicamentos pelos idosos é corroborada por estudos realizados no município de Fortaleza, Ceará, e em outros centros urbanos ${ }^{12,27}$.

Estudos sobre o uso de medicamentos genéricos em idosos tornam-se relevantes, como estratégia econômica e terapeuticamente eficaz para a saúde da população em geral, e principalmente daqueles que, como neste estudo, são detentores de um perfil socioeconômico mais fragilizado.
Apesar de os medicamentos serem a estratégia mais eficaz para o tratamento de doenças, o maior uso pela população idosa oferece muitas vezes uma relação risco-benefício desvantajosa e com repercussões negativas quanto ao estado de saúde e qualidade de vida deste grupo etário.

Atualmente os medicamentos potencialmente inapropriados e a elevada proporção de polifarmácia de medicamentos contínuos são dois dos maiores problemas concernentes à segurança do uso de medicamentos, sendo mais especificamente considerados fatores de risco para o desenvolvimento de eventos adversos as interações medicamentosas, hospitalização, qualidade de vida insuficiente e morte ${ }^{28}$. $\mathrm{O}$ uso de fármacos potencialmente inapropriados está relacionado também a problemas de saúde preveníveis, como depressão, constipação, imobilidade, confusão e riscos de fratura de quadril ${ }^{29,30}$.

Evidenciou-se que a presença de medicamentos de uso potencialmente inapropriados por parte dos idosos ocorreu por meio de medicamentos disponíveis no SUS. Acredita-se que o município seja orientado pela Relação de Medicamentos Essenciais (RENAME), mas verificou-se a presença de medicamentos não disponíveis na RENAME, necessitando uma readequação da assistência farmacêutica local, no sentido de prover a população de medicamentos eficazes e seguros, pois no campo da farmacoterapia o cenário que se vislumbra é o do dilema do "acesso ou excesso".

O grupo investigado possui grande dependência de medicamentos disponíveis gratuitamente pelo SUS, o que impõe aos gestores e profissionais de saúde maior responsabilidade quanto à saúde dos idosos. Esta grande demanda pelo serviço de farmácia pode proporcionar uma efetiva relação farmacêutico/paciente com o propósito de promover a saúde do idoso neste município.

Quanto à autoavaliação da qualidade de vida do idoso, deve-se reportar que este estudo não investigou a qualidade de vida dos idosos, mas a autopercepção desta no senso comum do idoso. A estreita relação 
com estudos sobre qualidade de vida anteriormente realizados, que se utilizaram de instrumentos psicométricos testados e validados, faz sugerir a utilização da autoavaliação da qualidade de vida como um indicador epidemiológico de fácil obtenção em estudos de grande base populacional, constituindo assim uma alternativa a instrumentos psicométricos longos e de difícil aplicação em grandes populações. Contudo, estudos posteriores com análise estatística mais complexa e com amostras diferenciadas em diferentes regiões devem ser realizados.

Neste estudo não se analisou a associação da polifarmácia de medicamentos contínuos e a utilização dos serviços de saúde, como número de consultas médicas e internações. O estudo analisou a prática da polifarmácia de medicamentos contínuos e a aquisição de medicamentos, demonstrando grande dependência da assistência farmacêutica do SUS em um município com Índice de Desenvolvimento Humano (IDH), em 2000, de 0,673, ocupando a $21^{\text {a }}$ posição no Estado do Ceará, e que 79,5\% dos serviços de saúde estão vinculados ao SUS ${ }^{31}$.
Este estudo apresentou algumas limitações, como a frequência das condições crônicas, superestimadas ou subestimadas, pois foram colhidas informações de usuários de medicamentos de uso contínuo, podendo sugerir um viés de seleção, memória ou pela ausência de diagnóstico confirmado. Contudo, o autorrelato é considerado uma medida robusta e de fácil obtenção de informações sobre as condições de saúde em inquérito domiciliar.

De acordo com o exposto ao longo deste estudo, a velhice deve ser entendida em uma perspectiva transdisciplinar e multiprofissional, resultado de um processo dialético social, demográfico, econômico e de saúde, relativa às necessidades fisiológicas, simbólico-culturais e estruturais da sociedade contemporânea, considerando suas particularidades geográficas e as desigualdades que essas impõem ${ }^{32}$.

Por fim, acredita-se ter contribuído com a produção de conhecimento científico pertinente ao campo da Saúde Coletiva, sobre as condições de vida e saúde da população idosa brasileira, particularmente a população do Nordeste semiárido.

\section{Referências}

1. Carvalho JAM, Garcia RA. O envelhecimento da população brasileira: um enfoque demográfico. Cad Saúde Pública 2003; 19(3): 725-33.

2. United Nations Population Division. World Population Ageing 2009. New York, NY, USA: United Nations; 2010.

3. Organização Mundial da Saúde. Grupo de Doenças Não-transmissíveis e Saúde Mental. Departamento de Prevenção de Doenças Não-transmissíveis e Promoção da Saúde. Envelhecimento e Curso de Vida. Madrid; 2002.

4. Alves LC et al. A influência das doenças crônicas na capacidade funcional dos idosos do Município de São Paulo, Brasil. Cad Saúde Pública 2007; 23(8): 1924-30.

5. Robert Wood Johnson Foundation. Partnership for Solutions Chronic Conditions: Making the Case for Ongoing Care; 2004.

6. Instituto Brasileiro de Geografia e Estatística. Síntese de indicadores sociais: uma análise das condições de vida da população brasileira; 2010.
7. Loyola Filho AI, Uchoa E, Firmo JOA, Lima-Costa MF. Estudo de base populacional sobre uso de medicamentos entre idosos: Projeto Bambuí. Cad Saúde Pública 2005; 21(2): 545-53.

8. Laroche ML, Charmes JP, Nouaille Y, Picard N, Merle L. Is inappropiate medication use a major cause of adverse drug in the elderly? Br J Clin Pharmacol 2006; 63(2): 17786.

9. Stewart RB, Cooper JW. Polipharmacy in the aged. Practical solutions. Drugs \& Aging 1994; 4(6): 449-61.

10. Hanlon JT, Fillenbaum GG, Burchett B, et al. Druguse patterns among black and nonblack community dwelling elderly. Ann Pharmacother 1992; 26: 679-85.

11. Roth MT, Ivet JL. Self-reported medication use in community-residing older adults: a pilot study. $A m \mathrm{~J}$ Geriatr Pharmacother 2005; 3: 196-204.

12. Secoli SR. Polifarmácia: interações e reações adversas no uso de medicamentos por idosos. Rev Bras Enfermagem 2010; 63(1): 136-40. 
13. Coelho-Filho JM, Marcopito LF, Castelo A. Perfil de utilização de medicamentos por idosos em área urbana do Nordeste do Brasil. Rev Saúde Pública 2004; 38(4): 557-64.

14. Ribeiro AQ et al. Inquérito sobre uso de medicamentos por idosos aposentados, Belo Horizonte-MG. Rev Saúde Pública 2008; 42(4): 724-32.

15. Instituto Brasileiro de Geografia e Estatística. Censo Demográfico 2000 e Pesquisa de Orçamentos Familiares POF 2002/2003. (2a ed.); 2004.

16. World Health Organization. The ATC Index with DDD System. Collaboration Centre for Drug Statistics Methodology. Institute of Public Health; updated revision Oslo: Norwegian, dec., 2009. Disponível em http://www.whocc.no/atcddd/ [Acessado em 05 outubro de 2009]

17. Fick MD, Cooper JW, Wade WE, Waller JL, Maclean R, Beers MH. Updating the Beers Criteria for potentially inappropriate medication use in older adults. Arch Intern Med 2003; 163(22): 2716-24.

18. Conselho Nacional de Saúde. Resolução $n^{\circ}$ 196/96 de 10 de outubro de 1996. Aprova as diretrizes e normas regulamentadoras de pesquisas envolvendo seres humanos. Brasília: Ministério da Saúde; 1996.

19. Haider SI, Johnell K, Thorslund M, Fastbom J. Analysis of the association between polypharmacy and socioeconomic position among elderly aged $>$ or $=77$ years in Sweden. Clin Ther 2008; 30(2): 419-27.

20. Martínez Querol C, Martínez VTP, Pérez MC, Viera JJL. Polifarmacia en los adultos mayores. Rev Cubana Med Gen Integr 2005; 21: 1-2.

21. Correa-de-Araújo R, Miller GE, Banthin JS, Trinh Y. Gender diferences in drug user and expenditures in a privately insured population of older adults. JWomen's Health 2005; 14(1): 73-81.

22. Loyola Filho AI, Uchoa E, Lima-Costa MF. Estudo epidemiológico de base populacional sobre uso de medicamentos entre idosos na Região Metropolitana de Belo Horizonte, Minas Gerais, Brasil. Cad Saúde Pública 2006; 22(12): 2657-67.
23. Galato D, Silva ES, Tiburcio LS. Estudo de utilização de medicamentos em idosos residentes em uma cidade do sul de Santa Catarina (Brasil): um olhar sobre a polimedicação. Ciência \& Saúde Coletiva 2010; 15(6): 2899-905.

24. Loyola Filho AI, Uchoa E, Firmo JOA, Lima-Costa MF. Influência da renda na associação entre disfunção cognitiva e polifarmácia: Projeto Bambuí. Rev Saúde Pública 2008; 42(1): 89-99.

25. Mastroeni MF, Erzinger GS, Mastroeni SSBS, da Silva NN, Marucci MFN. Perfil demográfico de idosos da cidade de Joinville, Santa Catarina: estudo de base domiciliar. Rev Bras Epidemiol 2007; 10(2): 190-201.

26. Pereira RS, Curioni CC, Veras R. Perfil demográfico da população idosa no Brasil e no Rio de Janeiro. Textos sobre Envelhecimento 2003; 6(1): 43-59.

27. Flores VB, Benvegnu LA. Perfil de utilização de medicamentos em idosos da zona urbana de Santa Rosa, Rio Grande do Sul, Brasil. Cad Saúde Pública 2008; 24(6): 1439-46.

28. Silvestre JA, Costa-Neto MM. Abordagem do idoso em programas de saúde da família. Cad Saúde Pública 2003; 19(3): 839-47.

29. Simonson W, Feinberg JL. Medication-related problems in the elderly: defining the issues and identifying solutions. Drugs Aging 2005; 22(7): 559-69.

30. Fick MD, et al. A randomized study to decrease the use of potentially inappropriate medications among community-dwelling older adults in a southeastern managed care organization. Am J Manag Care 2004; 10(11): 761-8.

31. Ceará. Perfil básico municipal: Quixadá. Instituto de Pesquisa e Estratégia Econômica do Ceará. Fortaleza-Ce. 2005.

32. Santos LM, et al. Grupo de promoção à saúde no desenvolvimento da autonomia, condições e saúde. Rev Saúde Pública 2006; 40(2): 346-52.

Recebido em: 11/04/2011

Versão final apresentada em: 17/10/2011 Aprovado em: 09/11/2011 